\title{
Topical Application of Terminalia chebula cream Inhibits Ultraviolet-B-Induced Psoriasis in Rats
}

Aruna Pai ${ }^{1 *}$, Jvln Seshagiri Rao ${ }^{2}$, Madhu J. Rajendra $^{3}$, Ashish S. Jain ${ }^{4}$

\begin{abstract}
Introduction: The present study aims at evaluating anti oxidant activity and inhibition of topical application of Terminalia chebula cream in ultraviolet -B-induced psoriasis in rats.

Materials and Methods: The seeds of Terminalia chebula were dried and powder was extracted with chloroform, ethyl acetate, ethanol, methanol, and deionized water by cold maceration method. Total phenolic content, total tannin content and total triterpenoid content was determined by different methods. Anti oxidant activity was evaluated by DPPH method and reducing power assay. Cream formulation of the optimized extract was prepared by hot fusion method. Psoriasis was induced by irradiatiating for 30 min at a vertical distance of $20 \mathrm{~cm}$ with UV light ( $385 \mathrm{~nm}$ ) on the skin. Severity index, hydroxyproline content and epidermal thickness was determined.

Results: Total Phenol (mg gallic acid/ g) content, Total Tannins (mg catechin/ g) content and Total triterpenoid (mg ursolic acid/ g) content was found to be significant for water extract.The reducing ability of water extract was always higher than methanol extract at all the concentrations.

Conclusion: We concluded that cream containing $0.1 \%$ water extract of Terminalia chebula seeds possess antioxidant activity along with potent antipsoriatic activity. Further, application of Terminalia chebula cream resulted in decrease in relative epidermal thickness of animal skin as well as other histopathological features. The present investigation aims at the development of potent phytomedicine for treatment of psoriasis from the Terminalia chebula plant.
\end{abstract}

Keywords: Anti oxidant activity, Anti psoriatic activity, Psoriasis, Terminalia chebula.

Journal of Applied Pharmaceutical Sciences and Research, (2020); DOI: 10.31069/japsr.v3i3.4

\section{INTRODUCTION}

Psoriasis is a chronic inflammatory skin disease with a strong genetic predisposition and autoimmune pathogenic traits. ${ }^{[1]}$ However, the causes of this disease are unknown, though interaction of genetic predisposition and external stimuli, including stress, infection, trauma, and drugs have been proposed.

Recent studies showed oxidative stress as one of the important factors in the pathogenesis of psoriasis. Increased reactive oxygen species (ROS) production and compromised function of antioxidant system was closely associated with psoriasis. ${ }^{[2]}$ Oxidative stress is an imbalance between oxidants and antioxidants, leading to a disruption of redox signaling and molecular damage. The skin is a potential target for oxidative injury, as it is continuously exposed to UV radiation and other environmental stresses generating ROS. ROSmediated oxidative damage involves a vast number of biological molecules since it causes lipid peroxidation, DNA modification, and secretion of inflammatory cytokines. A comprehensive and integrated antioxidant defense mechanism of the skin is vital in protecting it from ROS. However, excess ROS production or inadequate antioxidant protection creates a condition known as oxidative stress, contributing to the development of psoriasis.

With the understanding of factors behind pathogenesis of psoriasis, it could be presumed that a compound possessing antiinflammatory and/or antioxidant activity could be useful in the management of psoriasis. As a traditional folk medicine, Terminalia chebula (TC) has been widely used in Asian countries for its antibacterial, ${ }^{[3]}$ anti-inflammatory, ${ }^{[4]}$ and antioxidant activities. ${ }^{[5]}$

Previous studies showed anti-inflammatory activity of TC in lipopolysaccharide-stimulated RAW 264.7 cells in collagen-induced arthritis in a mice model and in Swiss albino mouse skin. Moreover, TC was shown to attenuate DMBA/croton oil-induced oxidative stress in skin of Swiss albino mouse. ${ }^{[6]}$ One of recent studies
${ }^{1}$ Asst .professor, Department of Pharmacognosy, SDDVCP\&RC,New Panvel ,Navi Mumbai, Maharashtra, India

${ }^{2}$ Professor, Department of Pharmaceutical Analysis, Yelamarthy college of pharmacy,Vishakapatnam, India

${ }^{3}$ AGM, Mylan Laboratories Limited,Anrich Inndustrial estate, Bollaram , Medak District, Hyderabad, India

${ }^{4}$ Principal, SDDVCP \& RC, New Panvel ,Navi Mumbai, Maharashtra, India Corresponding Author: Aruna Pai, Asst .professor, Department of Pharmacognosy, SDDVCP\&RC,New Panvel ,Navi Mumbai, Maharashtra, India, Email: arunapai78@yahoo.com

How to cite this article: Pai A, Rao JS, Rajendra MJ, Jain AS. Topical Application of Terminalia chebula cream Inhibits Ultraviolet-B-Induced Psoriasis in Rats. Journal of Applied Pharmaceutical Sciences and Research. 2020; 3(3):15-20

Source of support: Nil

Conflict of interest: None

showed that intragastrically administered TC not only suppresses inflammation, but also, suppresses keratinocyte proliferation and showed potent anti-psoriatic effects via heme oxygenase (HO)-1mediated inhibition of oxidative stress. ${ }^{[7]}$ The present study was designed to examine the effect of topical application of TC as a cream formulation on UV-B induced experimental rat model of psoriasis.

\section{Materials and Methods}

\section{Chemicals}

Seeds of TC were collected from Hyderabad's local areas and authenticated by the Botany Department of Osmania University (Letter number is required to be mentioned). Tretinoin $0.05 \%$ cream 
Topical Application of Terminalia chebula cream Inhibits Ultraviolet-B-Induced Psoriasis in Rats

(Janssen, UK) was purchased from a Pharmacy shop. Light liquid paraffin, soft paraffin, cetostearyl alcohol, and propylene glycol were purchased from SD. Fine Chemicals (India). All the solvents and chemicals used were of analytical grade and were purchased from Merck (India).

\section{TC Extraction}

The seeds of TC were shade dried and pulverized into fine powder using a grinder. Twenty-gram of the dried powder was each extracted three times with $50 \mathrm{~mL}$ of chloroform, ethyl acetate, ethanol, methanol, and deionized water by cold maceration method using glass percolator. The extracts were filtered, filtrates were pooled, and the solvent was removed at $40^{\circ} \mathrm{C}$, under reduced pressure using a rotary evaporator (Buchi, Switzerland). Finally, each extract was dried overnight using a vacuum drier before calculating the yield of each extract. All the dried extracts were stored at $-20^{\circ} \mathrm{C}$ before phytochemical composition analyses and antioxidant assays.

\section{Phytochemical Composition of TC Extracts}

\section{Total Phenolic Content}

Total phenolic content of the plant extracts was determined by the Folin-Ciocalteu assay. ${ }^{[8]}$ An aliquot of the extract (known concentration) was mixed with $5 \mathrm{~mL}$ Folin-Ciocalteu reagent (previously diluted with water 1:10 v/v) and $4 \mathrm{~mL}(75 \mathrm{~g} / \mathrm{l}$ ) of sodium carbonate. The tubes were vortexed for 20 seconds and allowed to stand for 30 minutes at $40^{\circ} \mathrm{C}$ for color development. Absorbance was read at $750 \mathrm{~nm}$ using a spectrophotometer (Beckman, USA). Extracts were evaluated at a final concentration of $1 \mathrm{mg} / \mathrm{mL}$. Total phenolic content was expressed as $\mathrm{mg} / \mathrm{g}$ gallic acid equivalent using the equation obtained from a calibration curve of gallic acid.

\section{Total Tannin Content}

Determination of condensed tannins was done using the procedure described by Sun et al. ${ }^{[9]}$ To $50 \mu \mathrm{L}$ of diluted sample, $3 \mathrm{~mL}$ of $4 \%$ vanillin solution in methanol and $1.5 \mathrm{~mL}$ of concentrated $\mathrm{HCl}$ were added. The mixture was allowed to stand for $15 \mathrm{~min}$, and absorption was measured at $500 \mathrm{~nm}$ against methanol as a blank. The amount of total condensed tannins is expressed as catechin equivalents $(\mathrm{mg} / \mathrm{g})$ using the equation based on the calibration curve.

\section{Total Triterpenoid Content}

Total triterpenoid content of the plant extracts were determined based on a coloring reaction involving vanillin-perchloric acid and glacial acetic acid with triterpenoids, as described by Chen et al., [10] with ursolic acid as the reference. Absorbance was read at $548 \mathrm{~nm}$, using a UV-visible-light recording spectrophotometer (UV-160 A, Shimadzu, Japan).

\section{Antioxidant activity of TC extracts}

\section{DPPH assay}

Free radical scavenging activity of TC extracts was measured by 1 , 1- diphenyl-2-picryl hydrazyl (DPPH). ${ }^{[11]}$ In brief, $0.1 \mathrm{mM}$ solution of DPPH in ethanol was prepared, and $1.0 \mathrm{~mL}$ of this solution was mixed with $1.0 \mathrm{~mL}$ of different concentrations $(0.01,0.05,0.1$, and $0.2 \%$ ) of the extract in ethanol. The reaction mixture was vortexed thoroughly and left in the dark at room temperature $27^{\circ} \mathrm{C}$ for $30 \mathrm{~min}$. The absorbance of the mixture was measured spectrophotometrically at $517 \mathrm{~nm}$. Ascorbic acid was used as a reference. Lower absorbance of the reaction mixture indicated higher free radical scavenging activity using the equation:

DPPH Scavanging $(\%)=1-A E / A D \times 100$

Where, $A E$ is the absorbance of the sample solution, and $A D$ is the absorbance of the blank DPPH solution.

\section{Reducing power assay}

The reducing power assay was carried out based on the method described previously (Yildrim et al. 2001). ${ }^{[12]}$ Briefly, $1.0 \mathrm{~mL}$ of plant extracts at different concentrations $(0.01,0.05,0.1$ and $0.2 \%)$ were mixed with $2.5 \mathrm{~mL}$ phosphate buffer $(0.2 \mathrm{M}, \mathrm{pH} 6.6)$ and $2.5 \mathrm{~mL}$ potassium ferricyanide $(10 \mathrm{~g} / \mathrm{L})$. The mixtures were then incubated at $50^{\circ} \mathrm{C}$ for 20 minutes. Then, $2.5 \mathrm{~mL}$ of trichloroacetic acid $(100 \mathrm{~g} / \mathrm{L})$ was added to the mixtures, which was then centrifuged at $3000 \mathrm{rpm}$ for 10 minutes. Finally, $2.5 \mathrm{~mL}$ of the supernatant solution was mixed with $2.5 \mathrm{~mL}$ of distilled water and $0.5 \mathrm{~mL} \mathrm{FeCl}_{3}(1 \mathrm{~g} / \mathrm{L})$, and absorbance was measured at $700 \mathrm{~nm}$ using a spectrophotometer (UV-VIS Shimadzu, Japan). Ascorbic acid was used as a standard reducing agent.

\section{In Vivo Studies}

\section{TC cream formulation}

TC cream formulation of the optimized extract was prepared by hot fusion method. Briefly, aqueous phase consisting of Optimized TC extract, disodium EDTA (0.01\%), butyl hydroxyl toluene $(0.001 \%)$, and dibasic potassium phosphate $(0.2 \%)$ was heated to $70 \pm 5^{\circ} \mathrm{C}$. Simultaneously, all oily phase components including light liquid paraffin (8\%), cetostearyl alcohol (10\%), propylene glycol (5\%), glycerin (5\%), white soft paraffin wax (12\%), polyethylene glycol 4000 (5\%), tween 80 (5.33\%), and butyl hydroxyl anisole $(0.001 \%)$ were also heated to $70 \pm 5^{\circ} \mathrm{C}$. Oily phase was then slowly added to the aqueous phase with continuous stirring and decreasing temperature resulting in the formation of o/w cream. The cream was stored in cool place until used. Placebo cream was prepared by the same method but without TC extract.

\section{Animals}

Wistar rat (weight: approx. 250-300 g) of either sex were used for the experiment. Animals were kept in the Shree Dhanvantary Pharmaceutical Analysis and Research Centre, Kim, Surat after approval from the Institutional Animal Ethical Committee (Reg. $1103 / a b c / 07 / C P C S E A)$ housed in polypropylene cages. Rice husk was used as the bedding material with a $12 \mathrm{~h}$ light-dark cycle, at temperature of $22 \pm 02^{\circ} \mathrm{C}$, humidity $30-70 \%$, and kept laboratory mouse pellet feed (Pranav Agro Ltd.). Pure drinking water was supplied ad libitum. The animals will be acclimatized to the laboratory conditions for a minimum period of seven days prior to commencement of treatment.

\section{Acute Dermal Toxicity Study}

The acute dermal toxicity test of final cream was determined according to the OECD guidelines No. 402 (Organization for Economic Corporation and Development). Approximately 24 hours prior to the test, hairs are removed from the dorsal area of the trunk of the test animals by shaving. Animals were divided into two groups of six animals each $(n=6)$. Group 1 animals were considered as control, and Group 2 animals received $2.5 \mathrm{~g}$ topically TCE (20.0\%) cream formulation (Target dose $2000 \mathrm{mg} / \mathrm{kg}$ ). The formulation should be held in contact with the skin with a porous gauze dressing and non-irritating tape throughout a 24-hour exposure period. The treated animals were monitored for 14 days for changes in fur, eyes, behavior, and toxic reactions. 
Topical Application of Terminalia chebula cream Inhibits Ultraviolet-B-Induced Psoriasis in Rats

\section{Evaluation of anti-psoriatic activity}

\section{Induction of psoriasis}

Hairs on the dorsal skin were removed by shaving. A total of $10 \%$ body surface area was irradiated for 30 minutes at a vertical distance of $20 \mathrm{~cm}$ with UV light $(385 \mathrm{~nm})$. After irradiation, rats were monitored for observing any change on the irradiated skin, any appearance of skin lesion, and other different behaviors. A total of 24 Wistar rats weighing were used for study. Psoriasis was induced in animals as mentioned above. After induction, animals were divided into four groups comprising of 6 animals $(n=6)$ in each group as follows:

Group 1: Normal control (psoriasis not induced; treated with vehicle) Group 2: Disease control (psoriasis induced; no treatment)

Group 3: Tretinoin group (psoriasis induced; treated with $0.05 \%$ tretinoin gel o.d. for 12 days)

Group 4: TCE cream group (psoriasis induced; treated with $0.1 \%$ TCE cream o.d. for 12 days)

\section{Severity Index $(S I)^{[13]}$}

Animals were evaluated by the severity index of psoriatic lesions every fourth day. A visual scoring system was developed based on severity. SI was scored on a scale from 0 to 3 where 0 represents no redness and erythema; 1 represents mild redness; 2 represents moderate redness and erythema; and 3 represents severe redness, erythema and scaling.

\section{Biochemical estimation ${ }^{[14]}$}

For the estimation of hydroxyproline content, the tissues were excised and store in formalin at $-20^{\circ} \mathrm{C}$. Tissue samples were hydrolyzed by treatment with $6 \mathrm{~N} \mathrm{HCl}$ for $3 \mathrm{~h}$ at $130^{\circ} \mathrm{C}$. The hydrolysate was neutralized to $\mathrm{pH} 7.0$ and was subjected to Chloramine-T oxidation for $20 \mathrm{~min}$. Five min after addition of oxidizer, $2.5 \mathrm{~mL}$ each of Ehrlich reagent was poured into every test tube, and they were immersed in a water bath at $60^{\circ} \mathrm{C}$. After $25 \mathrm{~min}$, the test tubes were transferred to an ice bath and, after cooling, 6.6 $\mathrm{mL}$ each of isopropyl alcohol was added. After thorough stirring, the samples were analyzed in the spectrophotometer, in $1 \mathrm{~cm}$ cuvettes, at a wavelength of $557 \mathrm{~nm}$, against a control, in which the solution being analyzed was replaced by water. The color developed practically does not change intensity for a period of 4 h. The hydroxyproline content in each sample analyzed was found from a calibration curve.

\section{Histopathology and epidermal thickness}

At the end of study, animals were anaesthetized using ketamine. Specimens of skin (tissues) were collected \& preserved in glass vials containing $10 \%$ formalin solution. Thicknesses of the stratum corneum and the cellular part of the epidermis were determined by light microscopic evaluation of skin biopsies from the lesion areas. For histopathology, longitudinal sections of skin specimen (about $5 \mathrm{~mm}$ thickness) were prepared by microtomy and stained with hematoxylin-eosin dye. The thickness of the cellular part of epidermis was determined using a calibrated ocular micrometer and all measurements were adjusted for magnification optics.

\section{Statistical Analysis}

Each phytochemical characteristic and antioxidant activity was determined in triplicate, using the same extract in order to determine reproducibility and to provide a mean \pm standard deviation (SD) using Microsoft Excel 2003. The LD50 value following the acute dermal toxicity test was determined using linear regression. All the in vivo data was represented as means \pm SD based on triplicate determinations. Data were analyzed for statistical significance using ANOVA, followed by Dunnett's multiple comparison test using GraphPad Prism 5 (GraphPad Software, Inc).

\section{Results and Discussion}

\section{Extraction Yield and Phytochemical Composition of TC Extract}

Table 1 presents the yield, and total phenolic, triterpenoid, and tannin content of the five extracts. The yield of the five extracts varied from $14.2 \pm 2.1 \%$ to $37.6 \pm 1.2 \%$. The increase in yield was observed with an increase in polarity $(\delta P)$ of the extracting solvents and followed the following ascending order: chloroform (2.7) < ethyl acetate (4.4) < ethanol (4.9) < methanol $(5.1)<$ deionized water (10.2). The maximum yield of $37.6 \pm 1.2 \%$ was observed for deionized water extract and the lowest yield of $14.2 \pm 2.1 \%$ for chloroform extract (Table 1). The results indicate the high polarity of major components of TC.

Total phenolic content was assayed using Folin-Ciocalteu reagent, which is routinely employed to study phenolic antioxidants. The method is fast, convenient, and reproducible. The total phenolic content of the extracts varied from $725.6 \pm 14.8$ to $989.5 \pm 15.2 \mathrm{mg}$ gallic acid/g extract with water extract demonstrating the highest phenolic content. The results show that the extracts are rich in phenolic contents. Phenolics have received considerable scientific attention because they are sources of potential natural antioxidants because of their ability to act both as efficient radical scavengers and metal chelators. ${ }^{[15]}$ Tannins are water-soluble high molecular weight phenolic compounds known to exhibit antioxidant and antimicrobial properties. Like phenolics, the highest tannin content was observed in water extract (39.8 $\pm 2.0 \mathrm{mg}$ catechin/ g extract) and lowest in chloroform extract ( $30.8 \pm 4.5 \mathrm{mg}$ catechin/g extract). This could be due to the water-soluble properties of tannins. The presence of tannins in the extract are indicative of their antioxidant efficacy. Another natural antioxidant, triterpenoid, was evaluated by colorimetry using ursolic acid as the standard. The water extract provided the lowest total triterpenoid content $(1.2 \pm 0.2 \mathrm{mg}$ ursolic acid/ $\mathrm{g}$ extract). The chloroform extract provided the highest triterpenoid content $(4.5 \pm 0.4 \mathrm{mg}$ ursolic acid/ g extract). The results showed lower triterpenoids in TC extracts. Nevertheless,

Table 1: Extraction yield and phytochemical contents of TC Extracts. Data as mean \pm SD.

\begin{tabular}{lllll}
\hline Extracts & Extraction yield & Total Phenol $(\mathrm{mg}$ gallic acid/g) & Total Tannins $(\mathrm{mg}$ catechin/g) & Total triterpenoid $(\mathrm{mg}$ ursolic acid/ $\mathrm{g})$ \\
\hline Chloroform & $14.2 \pm 2.1$ & $725.6 \pm 14.8$ & $30.8 \pm 4.5$ & $4.5 \pm 0.4$ \\
Ethyl acetate & $16.1 \pm 1.2$ & $795.9 \pm 12.7$ & $32.9 \pm 3.8$ & $4.0 \pm 1.0$ \\
Ethanol & $20.8 \pm 1.7$ & $850.5 \pm 10.8$ & $33.9 \pm 2.1$ & $3.2 \pm 0.8$ \\
Methanol & $25.9 \pm 1.8$ & $938.3 \pm 17.9$ & $34.5 \pm 1.5$ & $3.2 \pm 0.5$ \\
Water & $37.6 \pm 1.2$ & $989.5 \pm 15.2$ & $39.8 \pm 2.0$ & $1.2 \pm 0.2$ \\
\hline
\end{tabular}


Topical Application of Terminalia chebula cream Inhibits Ultraviolet-B-Induced Psoriasis in Rats

the presence of high content of phenol and tannins are indicative of therapeutic use of TC. In the present study, water and methanol extracts demonstrating higher yields and higher phenols and tannin contents were selected for further antioxidant evaluation.

Antioxidant properties of water and methanol extract of TC was evaluated using DPPH assay and reducing power assay. The DPPH assay measures the hydrogen-donating ability of watersoluble phenolics. Figure 1a shows the dose-response curve of DPPH radical scavenging activity of the TC extracts compared with ascorbic acid. The result shows that the water extract had an activity comparable to ascorbic acid. At the concentration of $0.1 \%$, the scavenging activity of water extract was $77.8 \pm 3.9 \%$, not significantly different from that of BHT (81.2 $\pm 3.8 \%)$, however, at the same concentration methanol extract had a scavenging activity of $65.3 \pm 5.5 \%$. From the results it was evident that the water extract, particularly at $0.1 \%$ concentration, showed proton-donating ability similar to that of ascorbic acid and this could serve as free radical inhibitors or scavengers, acting possibly as primary antioxidants. For confirmation of the antioxidant potential of TC extracts, reducing power assay was performed. Substances with reducing power indicate that they are electron donors and can reduce the oxidized intermediates of lipid peroxidation and act as primary or secondary antioxidants. ${ }^{[16]}$ In this assay, depending on the reducing power of each substance, the yellow color of the test solution changes to various shades of green and blue. The presence of reducers causes the conversion of the $\mathrm{Fe}^{3+} /$ ferricyanide complex to the ferrous form. By measuring the formation of Pearl's Prussian blue at 700 $\mathrm{nm}$, it is possible to determine the concentration of $\mathrm{Fe}^{3+}$ ion. The reducing power of water and methanol extract of TC in comparison to ascorbic acid as a function of their concentration is shown in Figure $1 \mathrm{~b}$. At $0.2 \%$ concentration, ascorbic acid demonstrated a higher reducing ability than the same water and methanol extract concentration. Interestingly, at $0.1 \%$ concentration, the reducing ability of water extract $(0.74 \pm 0.05)$ is similar to that of ascorbic acid $(0.76 \pm 0.05)$. Further, the water extract's reducing ability was always higher than methanol extract at all the concentrations, except at $0.01 \%$ concentrations where values for methanol extract were slightly higher than water extract. Thus, based on the results of the antioxidant activity, water extract at a concentration of $0.1 \%$ was selected for in vivo studies after formulating into cream base.
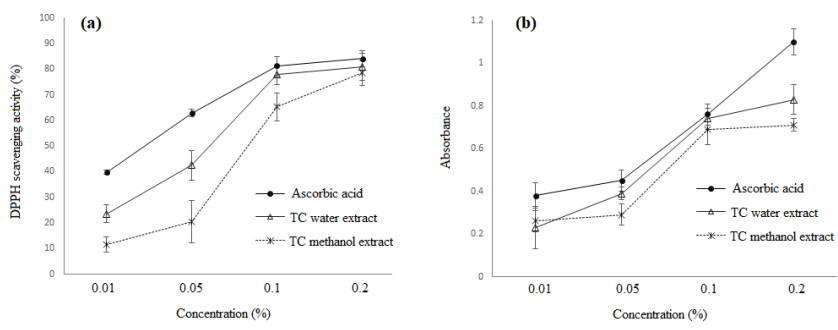

Figure 1: Anti oxidant activity

\section{In vivo Studies}

\section{Acute dermal toxicity}

The results of the acute dermal study showed that the TCE cream was non-toxic with $\mathrm{LD}_{50}>2000 \mathrm{mg} / \mathrm{kg}$. No toxic reactions, irritation, redness, inflammation and erythema was observed on skin of animals treated with $20 \%$ TCE cream. Further, no changes in fur, eyes, and behavior of animals was observed.

\section{Induction of psoriasis}

UV radiation-induced erythema and dermatitis in the rat is well-reported model for human psoriasis vulgaris. ${ }^{[17]}$ Exposure to UV radiation resulted in a sharply demarcated brownish-red lesion with scale formation along with microvascular dilatation, intraepidermal accumulation of polymorphonuclear leucocytes with microabscess and hyperproliferation of epidermal cells. All these etiologies are similar to those observed in psoriasis Vulgaris. In our studies, we found that exposure to UV radiation for $45 \mathrm{~min}$ resulted in development of psoriatic lesions with maximum severity index (Table 2). The second phase of erythema starts 6 hours after the irradiation and attained peak value between 24 and $48 \mathrm{~h}$. The color of the lesion was brownish-red with dark-brown scale. The development of other histological characteristics confirmed the development of experimentally induced psoriasis in rat model.

\section{Severity Index}

Severity Index was evaluated by visually observing the psoriatic lesions in each 4 days interval and then results are given in Table 2. From the results it was evident that TCE cream group and tretinoin group showed day to day decreases severity index. On day 4 , the tretinoin group treated $25.9 \%$ of psoriatic lesion while TCE cream group treated $18.5 \%$ as compared to the diseased group. On day 8 the percentage decrease in severity for the tretinoin group and TCE cream group was $33.3 \%$ and $27.8 \%$, respectively. On day 12 , tretinoin group completely treated (100\%) psoriatic lesion while TCE cream group treated $74.6 \%$ psoriatic lesions. The results demonstrated good anti-psoriatic property of the TCE cream.

\section{Hydroxyproline content}

In psoriatic lesions, the metabolisms of the collagen proteins undergo tremendous changes due to the acceleration of their turnovers as a result of inflammation. In few studies, it has been observed that prolidase activity is high in psoriatic lesions due to collagen deterioration. Further, high prolidase activity results in lowering of hydroxyproline content in psoriatic lesions and hydroxyproline serves as a biomarker for psoriasis. From the results (Figure 2) it is clearly evident that in the diseased group, levels of hydroxyproline $(13.9 \pm 0.21 \mu \mathrm{g} / 100 \mathrm{~g}$ tissue) are significantly less as compared to normal control group ( $19.8 \pm 0.9 \mu \mathrm{g} / 100 \mathrm{~g}$ tissue). The biochemical evaluation revealed that the hydroxyproline content

Table 2: Effect of topical formulations on severity index in UV radiation-induced psoriasis in rats

\begin{tabular}{|c|c|c|c|c|c|c|}
\hline \multirow[b]{2}{*}{ Groups } & \multicolumn{6}{|c|}{ Severity Index } \\
\hline & Day 4 & $\%$ decrease & Day 8 & $\%$ decrease & Day 12 & $\%$ decrease \\
\hline Normal & $0.0 \pm 0.0$ & - & $0.0 \pm 0.0$ & - & $0.0 \pm 0.0$ & - \\
\hline Diseased & $2.7 \pm 0.52$ & - & $1.8 \pm 0.41$ & - & $0.67 \pm 0.52$ & - \\
\hline Tretinoin & $2.0 \pm 0.0$ & 25.9 & $1.2 \pm 0.41$ & 33.3 & $0.0 \pm 0.0$ & 100 \\
\hline TCE cream & $2.2 \pm 0.41$ & 18.5 & $1.3 \pm 0.52$ & 27.8 & $0.17 \pm 0.41$ & 74.6 \\
\hline
\end{tabular}

Values are mean $\pm S D(n=6)$ 
Topical Application of Terminalia chebula cream Inhibits Ultraviolet-B-Induced Psoriasis in Rats

Table 3: Summary of histopathology and microscopic evaluation

\begin{tabular}{|c|c|c|c|c|}
\hline Group & Munro Microabscess & Elongation of rete ridges & Capillary loop dilatation & Epidermal thickness $(\mu m)$ \\
\hline Normal & - & - & - & 24.3 \\
\hline Diseased & + & +++ & +++ & 78.0 \\
\hline Tretinoin & - & - & - & 33.9 \\
\hline TCE cream & - & - & - & 35.0 \\
\hline
\end{tabular}

+ mild lesion; ++ moderate lesion; +++ severe lesion; - no lesion

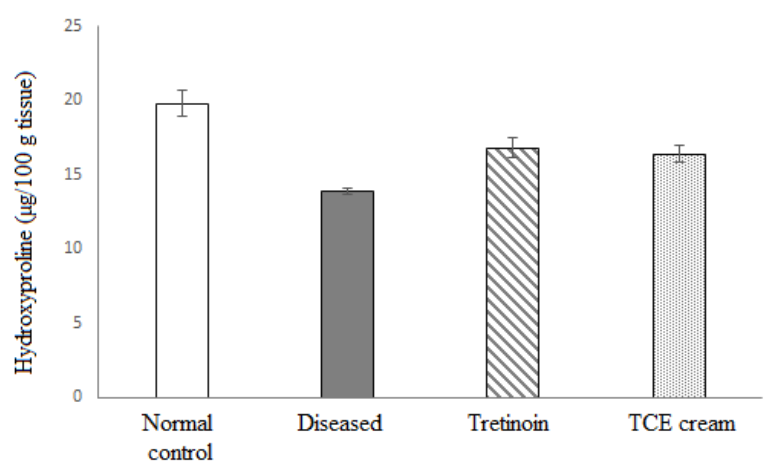

Figure 2: HP content

of both tretinoin ( $16.8 \pm 0.65 \mu \mathrm{g} / 100 \mathrm{~g}$ tissue) and TCE cream group $(16.4 \pm 0.53 \mu \mathrm{g} / 100 \mathrm{~g}$ tissue) are similar though the content is lower than normal control group indicating of collagen restoration and anti-inflammatory property of TCE cream.

\section{Histopathology and Epidermal thickness}

Results of histopathology and epidermal thickness of skin lesions are shown in Figure 3 and data represented in Table 3. In the case of the diseased group, the section showed regular elongation of rete ridges, Munro's microabscess, capillary loop dilation and a marked increase in relative epidermal thickness, all of which are etiological features of psoriatic lesions. Skin sections treated with tretinoin and TCE cream showed an absence of elongation of rete ridges, Munro's microabscess and capillary loop dilation (Figure 3).

The microscopy revealed reduction in epidermal thickness upon treatment. The epidermal thickness of the TCE cream group () was found to be significantly less than that of the diseased group () and not significantly different from the tretinoin group (). The results indicate good epidermis protecting property of TC extract. The results are indicative of the good anti-psoriatic property of TCE cream along with skin protective effects.

\section{ACKnowlwdgements}

Authors are thankful to the Management and Principal of Shri DDVCP\&RC, New Panvel for their support and timely help in carrying out the research activity.

\section{ConcLusion}

We concluded that TCE cream containing $0.1 \%$ water extract of Terminalia chebula seeds possess antioxidant activity along with potent anti-psoriatic activity. Further, application of TCE cream resulted in decrease in relative epidermal thickness of animal skin as well as other histopathological features. The present investigation aims at the development of potent phytomedicine for treatment of psoriasis from the Terminalia chebula plant.
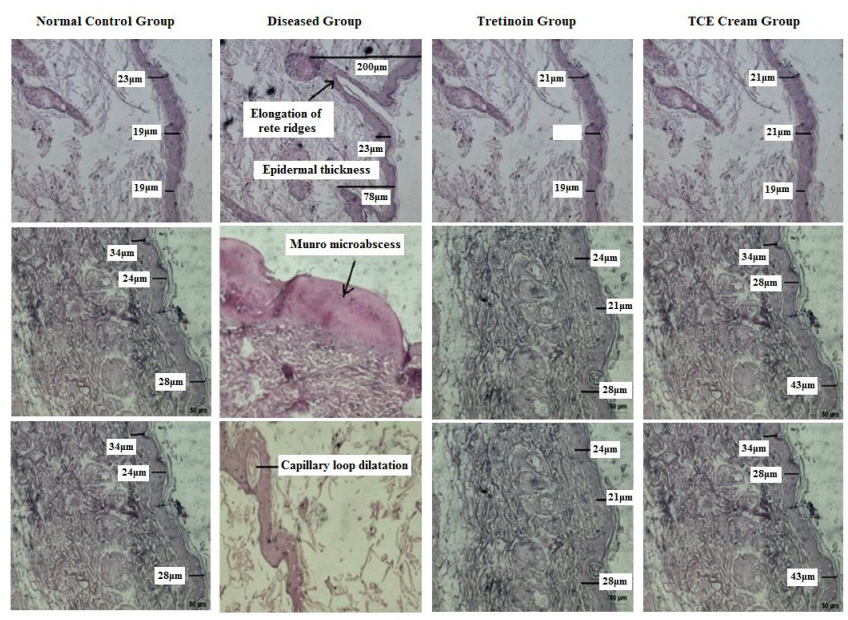

Figure 3: Histopathology

\section{References}

1. Adriana Rendon and Knut Schakel:,Psoriasis. Pathogenesis and Treatment International journal of molecular Sciences. 2019; 20(6): 1475.

2. Dipali P Kadam,Adinath N Suryakar,Rajesh D Ankush,Charushila $Y$ Kadam and Kishor H Deshpande.Role of Oxidative Stress in Various Stages of Psoriasis .International Journal of Clinical Biochemistry. 2010; 25(4): 388-392.

3. Kannan P, Ramadevi S.R and Waheeta Hopper .Antibacterial activity of Terminalia chebula fruit extract .African Journal of Microbiology Research. 2009;3(4) : 180-184

4. Md. Safkath Ibne Jami , Zakia Sultana, Md. Ershad Ali, Mst. Marium Begum, Md. Mominul Haque. Evaluation of Analgesic and AntiInflammatory Activities on Ethanolic Extract of Terminalia chebula Fruits in Experimental Animal Models. American Journal of Plant Sciences. 2014; 5(1): 63-69

5. Sarmistha Saha and Ramtej J Verma. Antioxidantactivity of polyphenolic extract of Terminalia chebula Retzius fruits. Journal of TaibahUniversity of Science. 2016;10(6): 805-812

6. Ferial Majed, Sana Nafees,Summya RashidNemat Ali, Syed Kazim Hasan, Rashid Ali,Ayaz Shahid and Sarwat Sultana. Terminalia Chebula Attenuates DMBA/Croton Oil-Induced Oxidative Stress and Inflammation in Swiss albino Mouse Skin. Toxicology International. 2015; 22(1): 21-29.

7. An J, Li T, Dong Y, Li Z, Huo J. Terminalia Chebulanin Attenuates Psoriatic Skin Lesion via Regulation of Heme Oxygenase-1. Cellular physiology and biochemistry, 2016; 39(2): 531-43

8. Fatima Alhakmani,Sokindra Kumar and Shah Alam Khan .Estimation of total phenolic content, in-vitro antioxidant and anti-inflammatory activity of flowers of Moringa oleifera. Asian Pacific journal of tropical biomedicine . 2013; 3(8): 623-627

9. B. Sun, J.M. Richardo-Da-Silvia, I.Critical factors of vanillin assay for catechins and proanthocyanidins. Journal of. Agriculture. Food Chemistry. 1998;46(10): 4267-4274

10. Mariia Shanaida. Determination of Triterpenoids in Some Lamiaceae Species. Research Journal of Pharmacy and Technology 2018; 11(7): 3113-3118

11. Chen HL, Lan XZ, Wu YY, Ou YW, Chen TC, Wu WT. The antioxidant activity and nitric oxide production of extracts obtained from 
the leaves of Chenopodium quinoa Willd BioMedicine. 2017; 7(4): 24.

12. Wan C, Yu Y, Zhou S, Liu W, Tian S, Cao S .Antioxidant activity and free radical-scavenging capacity of Gynura divaricata leaf extracts at different temperatures. Pharmacognosy magazine. 201; 7(25): 40-5

13. Srivastava AK, Nagar HK, Chandel HS, Ranawat MS. Antipsoriatic activity of ethanolic extract of Woodfordia fruticosa (L.) Kurz flowers in a novel in vivo screening model. Indian journal of pharmacology. 2016; 48(5): 531-536

14. Hemant K Nagar,Amit K srivastav,Rajnish Srivastav, Mahendra Ranawat. Evaluation of potent phytomedicine for treatment of psoriasis using UV radiation induced psoriasis in rats. Biomedicine \& Pharmacotherapy.
2016(4); 84: 2054

15. Deepak M Kasote,Surendra S Katyare, Mahabaleshwar V Hegde and Hanhong Bae .Significance of Antioxidant Potential of Plants and its Relevance to Therapeutic Applications. International journal of biological sciences. 2015; 11(8): 982-991

16. Ergul Belge Kurutas. The importance of antioxidants which play the role in cellular response against oxidative/nitrosative stress: current state.Nutrition Journal.2016; $15: 71$

17. A Vijayalakshmi and Geetha Madhira .Anti-psoriatic activity of flavonoids from Cassia tora leaves using the rat ultraviolet $\mathrm{B}$ ray photodermatitis model. Revista Brasileira de Farmacognosia . 2014 ; 24(3): 322-329. 